\title{
Prophylactic trypsin inhibition during the isolation procedure guarantees reproducible, high porcine islet yields
}

Heiser A, Ulrichs K, Müller-Ruchholtz W. Prophylactic trypsin inhibition during the isolation procedure guarantees reproducible, high porcine islet yields. Xenotransplantation 1994; 1:66-68. () Munksgaard, Copenhagen

Abstract: For isolating islets from the porcine pancreas, we established a semiautomated digestion method. Although the isolation technique was standardized and collagenase of controlled quality was used, until now the reproducibility of high islet yields was unsatisfactory. Our hypothesis was that pancreatic trypsin was responsible for this failure. The aim of this study was to investigate the effect of endogenous trypsin on islet yield. Our results demonstrate that a high trypsin level correlates with poor islet yield, whereas low trypsin activity always correlates with high islet yield.

Specific inhibition of trypsin results in low trypsin activity and

reproducible, high islet yields.

\section{Axel Heiser, Karin Ulrichs, and Wolfgang Múller-Ruchholtz}

Institute of Immunology, Medical School, University of Kiel, Kiel, Germany

Key words: islets of Langerhans xenogenic transplantation - swine enzyme activation - enzyme inhibitors

Address reprint requests to Axel Heiser, Institute of Immunology, Brunswiker Str. 4, D-24105 Kiel, Germany.

Accepted 5 April 1994

\section{Introduction}

For the treatment of diabetes mellitus type I, allogeneic transplantation of islets of Langerhans has become a reality during the past years. Because of comparable organ size, physiological similarity, and unlimited availability, the pig may serve as a suitable donor species for xenotransplantation of pancreatic islets. We used a standardized islet isolation technique based on the semi-automated digestionfiltration method [1]. This technique enabled us to isolate large amounts of islets from the porcine pancreas, but we were not satisfied because the yields varied considerably [2]. Our hypothesis was that the islet yield was influenced by the pancreatic enzymes. Therefore, the aim of this study was to investigate the effect of pancreatic trypsin during the isolation procedure and its effects on islet yield.

\section{Materials and methods}

Islet isolations were performed with pancreata from female pigs, which were always more than 2 years old and had a body weight between 200 and $250 \mathrm{~kg}$. Immediately after the splenic portion of the pancreas had been harvested, the organs were cannulated and transported in cold $\left(4^{\circ} \mathrm{C}\right)$ Eurocollins solution (Fresenius, Bad Homburg, Germany). The weight of the organs ranged from 15.0 to $99.7 \mathrm{~g}$. The isolation procedure was a modification of the semi-automated digestion-filtration method [1]. The collagenase solution, containing $1.8 \mathrm{PZ} \mathrm{U} / \mathrm{ml}$ collagenase (cat.-no. 17448, Serva, Heidelberg, Germany), was injected into the pancreas via the catheter. The digestion chamber was gently shaken by hand for $10 \mathrm{sec}$ every minute. Every other minute a sample of $150 \mu \mathrm{l}$ was taken, stained with $50 \mu \mathrm{l}$ dithizone solution and screened under a microscope [3]. The dithizone solution was prepared with $50 \mathrm{mg}$ dithizone (Sigma, Germany) in $5 \mathrm{ml}$ dimethylsulfoxide diluted 1:20 with HBSS, supplemented with 5\% fetal calf serum (Conco, Germany). The elution of the digested tissue was started when a significant number of well digested islets were observed (after $21.5 \pm 5.42 \mathrm{~min}$ ). After sedimentation $\left(270 \times g, 4 \mathrm{~min}, 4^{\circ} \mathrm{C}, 2 \times\right)$, islet yield was quantitated. Only islets with a diameter $\geq 50 \mu \mathrm{m}$ and without a detectable exocrine rim were counted with the help of the islet specific dye dithizone, and the islet yield was calculated as islets/g of organ. We have been using dithizone for many years, and in our hands dithizone always led to reproducible results as long as we followed exactly the above procedure (this is in accordance to personal information from other groups and to all available evidence from the 
literature that properly done dithizone staining does not lead to false-positive or -negative results, regarding cells within the pancreas). Variations in the trypsin concentrations did not influence the reliability of the dithizone staining. The viability was determined by staining the isolated islets with fluorescein diacetate and propidium iodide (FDA/ PI). Samples of the collagenase solution were collected before injection into the organ and at the end of the recirculation period and collagenase and trypsin activity were measured. The collagenase activity assay was performed using PZ (4-[phenylazobenzyl-oxycarbonyl]-L-prolyl-L-leucyl-glycyl-Lprolyl-D-arginine; Serva, Heidelberg, Germany) as substrate [4] and trypsin activity was evaluated using BAEE (N $\alpha$-benzoyl-L-arginine ethyl-ester-HCL; Serva, Heidelberg, Germany) as substrate, following the standard protocol of Boehringer Mannheim. In order to inhibit trypsin the collagenase solution was supplemented with $1.0 \mathrm{mM}$ Pefabloc (4-(2aminoethyl)-benzenesulfonyl fluoride hydrochloride; Boehringer, Mannheim, Germany).

\section{Results}

Twelve isolations were performed with the standardized isolation procedure. The enzyme activity developed as follows: collagenase activity decreased from $1.82 \pm 0.22$ to $1.22 \pm 0.19 \mathrm{PZ} \mathrm{U} / \mathrm{ml}$. Unexpectedly, trypsin activity showed two different types of kinetics. Before injection the activity was $0.31 \pm 0.12$ BAEE U/ml. In eight cases it increased slightly to $0.57 \pm 0.31 \mathrm{BAEE} \mathrm{U} / \mathrm{ml}$ and in four other cases it increased very strongly to $3.34 \pm 0.27 \mathrm{BAEE} \mathrm{U/ml}$ (y-axis in Fig. 1).

Because of the great variations in the islet yield (between 0 and 12,491 islets/g of organ) after the above isolations ( $\mathrm{x}$-axis in Fig. 1), we looked for a correlation between islet yield and enzyme activity: In the group of isolations with low trypsin activity $(\leq 1.50 \mathrm{BAEE} \mathrm{U} / \mathrm{ml})$ a high islet yield of $6,795 \pm$ 3,697 islets/g of organ was found, whereas only a poor islet yield of $507 \pm 739$ islets/g of organ was observed in the group with high trypsin activity (>1.50 BAEE U/ml). Standard deviations of the means do not overlap, thus the differences are significant. The enzymes clostripain and neutral protease, which were also tested (data not shown), showed no influence on the islet yield. In all cases the islet mass, i.e., only islets without an exocrine rim, was 5 to $10 \%$ within the digests. The isolations performed with collagenase solution supplemented with the trypsin inhibitor Prefabloc (Fig. 1) showed only a slight increase in trypsin activity (from 0.17 \pm 0.09 to $0.35 \pm 0.15 \mathrm{BAEE} \mathrm{U} / \mathrm{ml}$ ) and the islet yield was very satisfactory $(8,395 \pm 3,533$ islets/g of organ) in comparison to the isolations with naturally high trypsin activity. Taken together, it becomes evident that low trypsin activity $(n=11)$ ensures a high islet yield.

\section{Discussion}

The decrease in collagenase activity during the isolation procedure may be caused by 1) binding of collagenase to the donor tissue, which was separated from the samples before the start of the activity test and 2) proteolytic damage to collagenase molecules. When determining the collagenase concentration these effects should be considered. A concentration of $1.80 \mathrm{PZ} \mathrm{U}$ collagenase/ml worked very well in the present study.

\section{Post-Isolation Trypsin Activity [U/ml]}

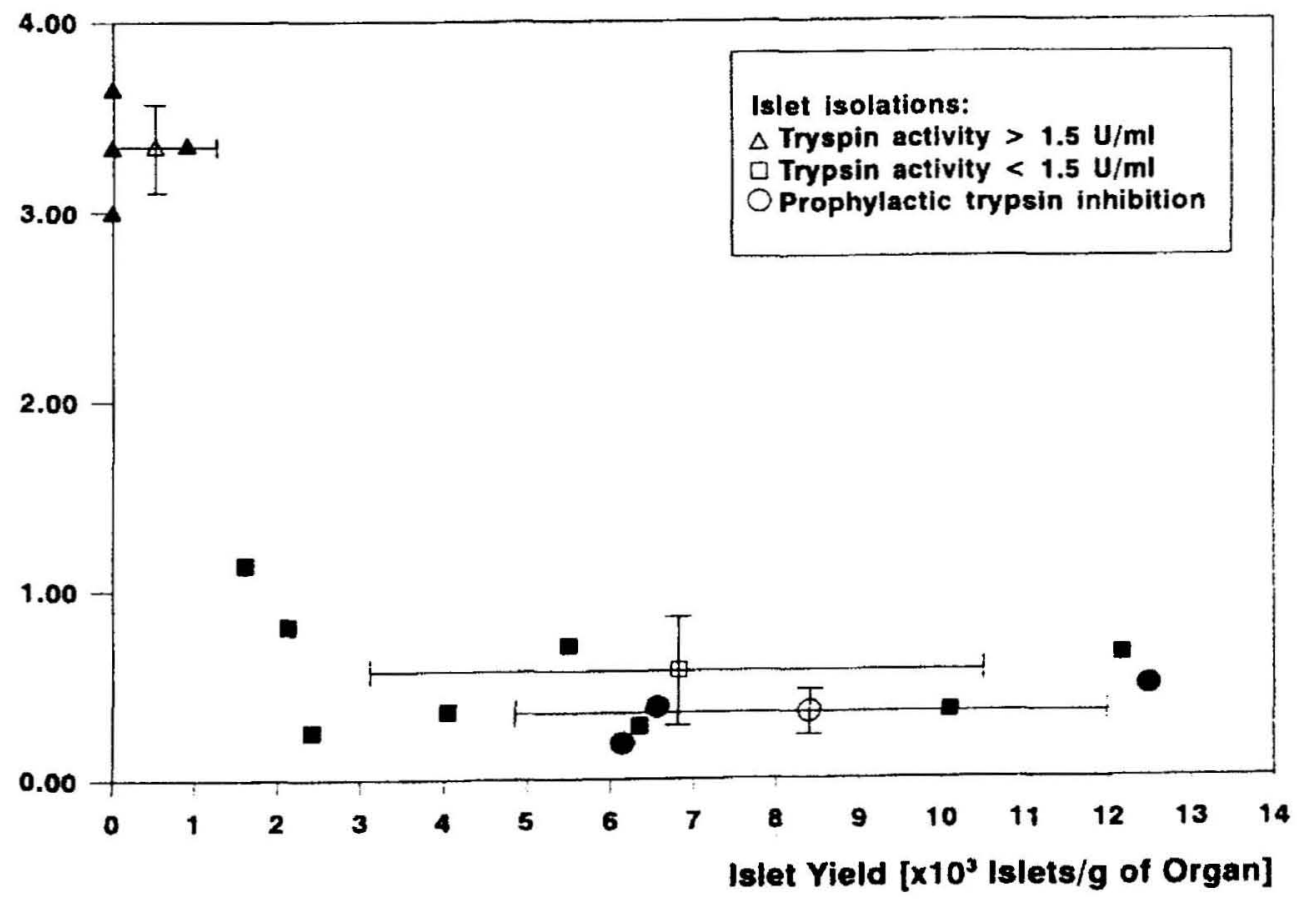

Fig. 1. Correlation of trypsin activity and islet yield. The figure shows the results of trypsin activity tests and islet yields. Three groups of isolations are shown; $\mathbf{n}$, isolations with trypsin activity <1.5 BAEE-U/ $\mathrm{ml}(n=8) ; \quad \boldsymbol{\Delta}$. isolations with trypsin activity $>1.5$ BAEE-U/ $\mathrm{ml}(n=4)$; and $\bullet$, isolations with trypsin inhibition by Pefabloc $(n=3)$. Open signs represent the means \pm standard deviations, closed signs document the single experiments. 


\section{Heiser et al.}

To achieve consistently high islet yields is the major problem in isolating islets from both the porcine and the human pancreas. In previous studies the influence of pig age $[2,6]$, pig race $[2,7]$, and $\mathrm{pH}$ of the isolation medium [2] on the islet yield was shown. We standardized the isolation technique accordingly, but we still did not achieve satisfactorily reproducible, high islet yields. That led us to look into the enzymatic digestion process itself.

An increase in proteolytic activity during the isolation procedure of islets of various species was described by Wolters et al. [5]. In this study we confirmed these findings for porcine islets and extended them by determining that particularly trypsin was released during the isolation procedure, presumably due to the disintegration of the exocrine tissue. The combination of exogenous collagenase and endogenous pancreatic trypsin may easily initiate an unspecific, fast, and uncontrollable tissue disintegration process.

For the first time this study shows that naturally high trypsin activity ( $>1.50 \mathrm{BAEE} \mathrm{U/ml)} \mathrm{prevents}$ the release of large numbers of islets. The failure of islet isolations was apparently caused by the individual high trypsin activity of porcine pancreata. Such factors as pig age $[2,6]$, pig race $[2,7]$, nutrition [8], and retrieval procedure [9] may directly influence the trypsin content of the pancreas. Indirectly, via trypsin activity during the isolation procedure, they may also affect the islet yield. Because of the activation of trypsinogen by trypsin and other proteolytic enzymes [10], the proteolytic activity of the collagenase preparation may possibly have an additional effect. Our studies indicated a lack of an apparent role of clostripain and neutral protease. Their kinetics showed no individual variations and no interaction with the isolation procedure.

Since it can not been foreseen which individual donor organ develops high trypsin activity during the isolation procedure, the aim must be to guarantee low trypsin activity. This may be achieved by supplementing the collagenase solution with $10 \%$ bovine serum albumin (BSA) as protease inhibitor [5]. A positive effect $(+48 \%)$ on rat islet yield was described, but in our opinion BSA has three disadvantages: it is only a competitive and unspecific inhibitor; considering own experiments (data not shown), it obviously inhibits collagenase and it interferes with the activity tests. In contrast, Pefabloc appears to be a much more reliable trypsin inhibitor-it inhibits trypsin irreversibly and specifically, it does not inhibit collagenase, and it does not interfere with the activity tests. All isolations performed with Pefabloc resulted in low trypsin activity and a high islet yield. Since the islet isolation technique requires the preparation of the whole pancreas, the natural trypsin content of the donor organs would not be determined in this particular group. However, this was of little importance since we intended to prophylactically downregulate the development of high trypsin activity with Pefabloc. To ensure that the pancreas is completely disintegrated at the proper time a small but well-controllable degree of proteolytic activity may be necessary. The role of residual trypsin activity awaits further investigation.

The physiological similarity between the porcine and human pancreas and the similarity of the technical problems encountered in isolating porcine and human islets will probably allow us to transfer the above knowledge from porcine to human islet isolation. Prophylactic and specific trypsin inhibition during islet isolation may have a comparably positive effect on human islets.

\section{References}

1. Ricordi C, Socci C, Davalli AM, et al. Isolation of the elusive pig islet. Surgery 1990:107:688.

2. Heiser A, Ulrichs K, Müller-Rlchholtz W. Influence of porcine race, age and $\mathrm{pH}$ of the isolation medium on porcine pancreatic islet isolation success. Transplant Proc 1994:26:618.

3. Latif ZA, Noel J, Alejandro R. A simple staining of fresh and cultured islets. Transplantation 1988:45:827.

4. WüNSCH E, HEIDRICH HG. Zur quantitativen Bestimmung der Kollagenase.Hoppe-Seyler's Z Physiol Chem 1963:333:149.

5. Wolters GHJ, Suylichem PTR, van Deinnen JHM, van SCHILFGARDE R. Increased islet yield by improved pancreatic tissue dissociation: The effects of bovine serum albumin and calcium. Transplant Proc 1989:21:2626.

6. Socci C, Ricordi C, Davalli AM, et al. Selection of donors significantly improves pig islet isolation yield. Horm Metabol Res 1990:25:32.

7. Ulrichs K, Bosse M, WACKer $\mathrm{HH}$, et al. Histological analysis of the porcine pancreas to improve islet yield and integrity after collagenase digestion. Transplant Proc 1994:26:610.

8. LinN T, Schmitz P, KLOER HU, et al. Experimental islet isolation from donors with different nutrition regimens. Horm Metabol Res 1990:25:17.

9. Ricordi C, Socci C, Davalli AM, et al. Effect of pancreas retrieval procedure on islet isolation in the swine. Transplant Proc 1990:22:442.

10. McShane P, Sutton R, Gray DWR, Morris PJ. Techniques for islet preparation: Protease activity in pancreas islet isolation by enzymatic digestion: Diabetes 1989:38(Suppl. 1): 126 . 